\title{
Synchronization of RFID Readers for Dense RFID Reader Environments
}

\author{
Kin Seong Leong, Mun Leng Ng, Member, IEEE, Alfio R. Grasso, Peter H. Cole \\ Auto-ID Laboratory, School of Electrical and Electronic Engineering, the University of Adelaide \\ \{kleong, mng, alf, cole\}@eleceng.adelaide.edu.au
}

\begin{abstract}
For a dense RFID reader deployment, such as in warehouse RFID deployment, where hundreds of antennas will be positioned in a building, the interference between all these readers must be studied carefully to avoid severe reader collisions. Strict RFID regulations and standards have been imposed, trying to address the problem of reader collision and also the problem of RFID devices interfering with other devices operating in nearby frequency bands. However, these guidelines and regulations are not entirely friendly for dense RFID reader deployment, in some cases it is not possible to have a feasible RFID system while adhering to these regulations. Hence, this paper proposes the synchronization of RFID readers, to enable successful dense RFID reader deployment. A case study targeted at European operations is presented in this paper to visualise the actual synchronization of RFID readers in real life applications. Some fine-tuning methods are also suggested to further improve the performance of readers in a high reader density population area.
\end{abstract}

\section{Introduction}

Radio Frequency Identification (RFID) has received much attention recently as it has been widely believed that RFID can bring revolution to supply chain management, replacing barcodes as the main object tracking system. Several major supply chain companies, such as Wal-Mart in the USA, have deployed RFID systems in some of their supply chains [1]. Initial test runs of RFID deployment show encouraging results [2], and hence large scale RFID deployment is planned. However, before any successful deployment can be achieved, some RFID issues have to be resolved. One of them is the RFID reader collision problem, which is the focus of this paper.

The term "reader collision(s)" is discussed extensively in [3], [4]. In this paper, reader collision is simply defined as the phenomenon where an interrogation signal from a certain reader disrupts the communication between a tag and another reader, and this reader collision problem is potentially magnified in a dense reader environment, such as a warehouse. Various regulatory bodies and standardization bodies have tried to regulate the operations of RFID readers. In this paper, the ETSI 302208 as introduced by the European regulatory body and the EPC Class 1 Generation 2 as recommended by EPCglobal are used as the basis of RFID reader regulations. However, as will be discussed in more detail in the later part of this paper, the restrictions that are put on the operation of RFID readers are very strict and in a way it is quite impossible to have an uncoordinated large scale deployment of RFID readers. Hence, this paper introduces the idea of RFID reader synchronization, to enable good RFID performance in dense reader environment, while adhering to strict regulations.

The next section introduces the ETSI 302208 and EPC Class 1 Generation 2 Protocol and their impact on RFID reader deployment. Section 3 explains the concept of RFID reader synchronization and how it adheres to strict regulations. A case study on RFID reader placement is presented in Section 4. Ways of fine-tuning RFID reader positioning is discussed in Section 5, followed by conclusion in Section 6 .

\section{Background}

\subsection{ETSI 302208}

ETSI 302208 is a European regulation governing the operation of RFID readers. It allocates the frequency band of 865 to $868 \mathrm{MHz}$ for RFID deployment. This frequency band is then divided into 15 sub-bands or channels, each spans a total of 200 $\mathrm{kHz}$. However, when a reader is operating at the maximum radiated power, which is $2 \mathrm{~W}$ ERP (Effective Radiated Power), only 10 sub-bands are available, while the remaining 5 are utilized as guard bands. ETSI 302208 also introduces the concept of 
"Listen Before Talk". An extract from the ETSI 302 208 best describes the essence of "Listen Before Talk". It says "Prior to Transmission, the interrogator must listen for the presence of another signal within its intended sub-band of transmission. The listen time shall comprise a fixed period of $5 \mathrm{~ms}$ plus a random time of $0 \mathrm{~ms}$ to $5 \mathrm{~ms}$ in 11 steps. If the sub-band is free the random time shall be set to $0 \mathrm{~ms}$ " [5]. The threshold to determine the presence of another signal within the intended sub-band is shown in Table 1 below.

Furthermore, once a sub-band has been selected, the RFID reader is permitted to use that sub-band for up to $4 \mathrm{~s}$. After use, it must free the sub-band for at least 100 ms. A reader can however, listen to another sub-band for $5 \mathrm{~ms}$ and if free use that new sub-band.

Table 1. Transmit and threshold power

\begin{tabular}{|c|c|c|}
\hline ERP (W) & ERP (dBW) & Threshold (dBW) \\
\hline Up to 0.1 & Up to -10 & $\leq-113$ \\
\hline 0.1 to 0.5 & -10 to -3 & $\leq-120$ \\
\hline 0.5 to 2.0 & -3 to 3 & $\leq-126$ \\
\hline
\end{tabular}

\subsection{EPC Class 1 Generation 2 Protocol}

"EPC Radio-frequency Identification Protocols Class 1 Generation 2 UHF RFID Protocol for Communication at $860 \mathrm{MHz}-960 \mathrm{MHz}$ " [6], in short EPC $\mathrm{C} 1 \mathrm{G} 2$, is the standard protocol developed by EPCglobal for RFID devices for use within the supply chain. This protocol outlines the air interfaces and commands between an RFID reader and an RFID tag. It also includes the spectrum management of RFID operation in a dense reader environment. Frequency hopping or frequency agile systems are the suggested techniques. An allocated frequency band, as allowed by local regulatory body, is divided into sub-bands or channels. A reader will only use a certain channel for communication, not the entire allocated frequency band. Also, according to EPC C1G2, for dense reader operation it is recommended that interrogator transmissions operate in even-numbered channels and tag backscatters are located in odd-numbered channels.

\subsection{Problem in dense reader environment}

With the implementation of ETSI 302208 and EPC $\mathrm{C} 1 \mathrm{G} 2$, it was discovered that when a reader is operating at a certain sub-band or channel, this reader will effectively prevent other readers from using that channel within an unacceptable large area. [5] and [6] have presented detailed discussions and analysis on this matter and Table 2 below summarises the minimum distance between two antennas connected to readers before one antenna operating at a certain channel will allow the other antenna from using that channel.

Table 2. Min. Distance Between Antennas

\begin{tabular}{|c|c|c|c|}
\hline Channel & \multicolumn{3}{|c|}{ Antenna Projecting Horizontally } \\
\cline { 2 - 4 } Difference & Front $(\mathrm{m})$ & Side $(\mathrm{m})$ & Back $(\mathrm{m})$ \\
\hline 0 & 1400 & 350 & 210 \\
\hline 1 & 180 & 45 & 30 \\
\hline 2 & 130 & 25 & 15 \\
\hline 3 & 95 & 20 & 10 \\
\hline
\end{tabular}

\section{Reader synchronization}

Under the concept of reader synchronization, all the RFID readers in a certain area, for example all the readers in a warehouse, are networked together through a central control unit. The connection method can be the common Ethernet connection, or equivalent.

Since all the readers are linked together, they can be directed to carry commands at a same time. Also, they can be assigned channels dynamically, so that the spectrum management is optimised while the reader collision is minimised.

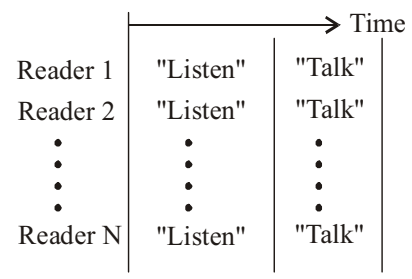

Figure 1. Synchronization of all readers: All the readers start to "Listen" at a same time and finish "Listen" at a same time too.

European regulation allows 10 channels when maximum radiated power, $2 \mathrm{~W}$ ERP, is used. Following the recommendation of EPC C1G2, 5 of them, the even-numbered channels, are used for reader interrogation. All the readers are "Listen Before Talk" compatible. They are configured to start to "Listen" at the same time, and then at the end of the listen period, they can all synchronously start to "Talk", as shown in Fig 1 . This is due to the fact that according to ETSI 302208 , if there is no signal detected in the intended channel of interest, the "Listen" time is fixed. Hence, all the readers, which start "Listening" at the same time, will start "Talking" at the same time. If a reader is turned on at a different time, or if a reader loses synchronization that reader will start again in synchronism with the rest of the readers, after the last reader has finished its "Talk" session.

\section{Case study}




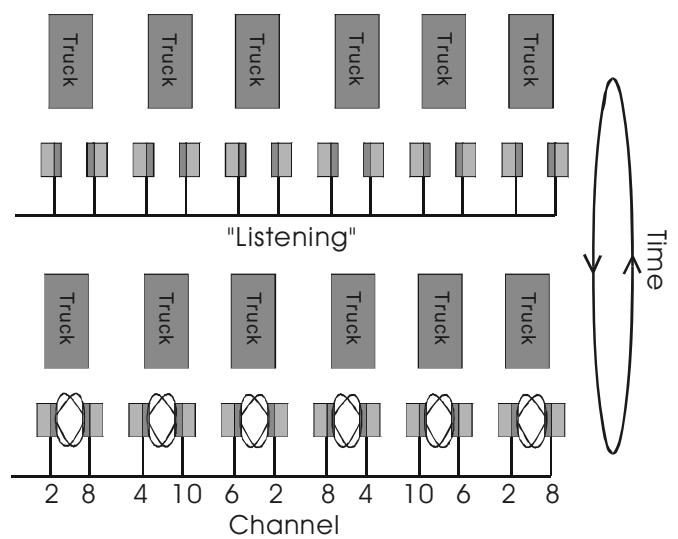

Figure 2. Alternating of "Listening" and "Talking" mode.

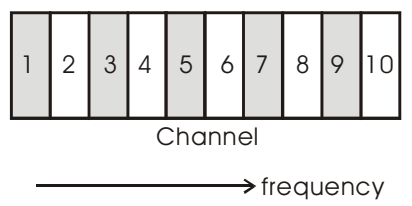

Figure 3. Channelling of the allocated frequency spectrum.

A case study on dense RFID reader deployment at the dock doors of a warehouse is presented here. As shown in Fig. 2, the dark colour rectangles represent trucks loading or unloading goods at the dock doors of a warehouse. Each door is around $3 \mathrm{~m}$ in width, and has two RFID antennas facing each other for tag interrogation.

Since all the readers are synchronised in a way described in Section 3, they will start "Listening" at a same time and will be assigned channels for interrogation at the end of "Listen" period. The assignment of channels will be geographically influenced. Two readers assigned to be operating in a same channel will be as far apart as possible. Also, the neighbouring antennas will be using channels as far apart as possible. As illustrated in Fig. 3, the spectrum is split into 10 channels, all 5 of the odd-numbered channels are reserved for tag backscattering while all 5 of the even-numbered channels are assigned for reader interrogation. Fig. 2 shows how the channel assignment is done. The antenna on the furthest left is using channel 2 for interrogation. The next antenna on its immediate right is using channel 8 , which is 6 channels away. Channel 10, though is the furthest channel away, is not chosen. This is because the arrangement of $[2,8,4,10,6]$ gives best channel separation between every channel.

\section{Synchronized RFID system fine-tuning}

Fine-tuning can be carried out to further reduce the tendency of reader collision, to enable more reader deployments at the same time, so that the RFID coverage in the area can be increased.

\subsection{Reduction of output power}

Although up to $2 \mathrm{~W}$ ERP can be used in single or small population reader environment, in dense reader populations this higher power may not be necessary. Currently, a state of the art reader can read up to $10 \mathrm{~m}$. However, normal reading operation does not require such a read range. In the case study presented in Section 4, the dock doors of the warehouse are around $3 \mathrm{~m}$ in width. Since two antennas are positioned facing each other in every dock door, the read range required is also around $1.5 \sim 2 \mathrm{~m}$. By reducing the radiated power of readers, the minimum distance between two antennas using a same channel can also be reduced, which is beneficial in a dense reader environment.

\subsection{Reduction of overall reader talking time}

While it is possible to talk for 4 seconds, readers and applications should be configured to talk for only the time necessary to capture tag data. There is no optimum talking time. It all depends on the type of application and also the surroundings of the deployment zone. On-site fine-tuning and measurement is needed before the reduction of talking time can be carried out.

\subsection{Use of external sensors}

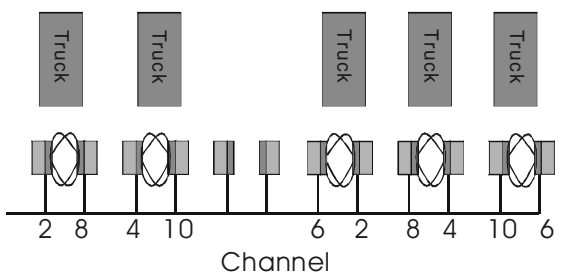

Figure 4. Using sensors in RFID system. Both the antennas at dock door 3 are switched off when the absence of truck 3 is detected.

Sensors can be used to turn RFID reader on only when tags are approaching to further reduce reader interference in that area. This will free up the channels allocated for those antennas, and also to avoid unnecessary interference to other surroundings reader antennas. For example, external sensors can be attached to the dock door in the case study in Section 4. When the dock door is not in use, the designated RFID readers would be switched off, as shown in Fig 4. Optionally, the central control unit can then 
dynamically shift the channels assigned for the antennas at door 3 to door 4 .

\subsection{RF opaque or RF absorbing materials}

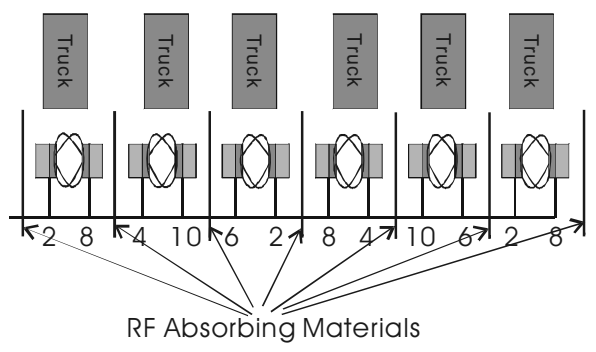

Figure 5. Use of RF absorbing materials. The antennas facing each other at the same door is at least 4 channels away.

Another effective, but more expensive, way to reduce readers interference and collision, is to utilize RF opaque or RF absorbing materials to contain the interrogating signal within the designated zone of interrogation. For the case study presented in Section 3 , the use of such materials is shown in Fig. 5. Although there will still some signal leakage through the door openings, it would not have caused much interference. This is due to the fact that the signal strengths at the sides of the antenna are relatively weak as compared to the front of the antenna. According to [6], the gain at the side of a typical RFID antenna is approximately $20 \mathrm{~dB}$ less than the gain at the front of the antenna.

\subsection{Switching of channels}

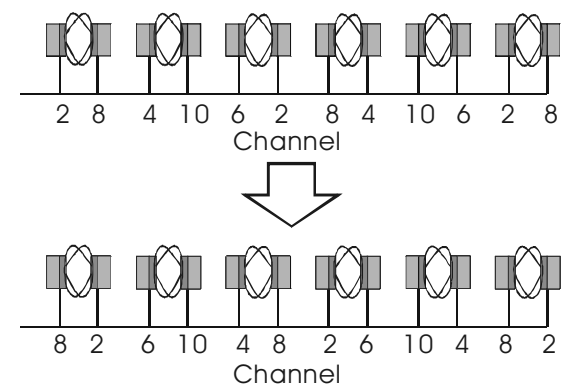

Figure 6. Channel switching within antennas.

Interrogating Channels can be switched around every cycle of "Listen Before Talk". This is to prevent the jamming of the interrogation signal by any external noise. Fig. 6 shows a simple example on how the switching is done. There are other more complex switching methods involving higher artificial intelligence in the central control unit, depending on the noise received from the surrounding environment.

\section{Conclusion}

This paper has identified synchronization of RFID readers as a mechanism to assist in RFID readers deployment in dense reader environments. Several fine-tuning methods are also presented in optimising the performance of a synchronised RFID system. As compared to conventional unsynchronised RFID systems, a synchronised RFID system can offer more coverage, less reader collision or interference, while strictly following the European regulations and the EPC $\mathrm{C} 1 \mathrm{G} 2$ recommendation. However, these benefits require the use of more complex hardware and hence can marginally increase deployment costs. Reader synchronization has not been tested in real situation, and hence will require future study in this area.

\section{Reference}

[1] D. Barlas, "Wal-Mart's RFID mandate," 4 June 2003.[Online]. Available: http://www.line56.com/articles/ default.asp?ArticleID=4710.

[2] J. Rendon, "Wal-Mart touts RFID results," 18 Jan 2005. [Online]. Available: http://searchmobilecompu ting.techtarget.com/originalContent/0,289142,sid40_gci1048 680,00.html?bucket=NEWS

[3] D. W. Engels, S. E. Sarma, "The reader collision problem," IEEE International Conference on Systems, Man and Cybernetics, 6-9 Oct. 2002.

[4] B. Carbunar, M. K. Ramanathan, M. Koyuturk, et al, "Redundant reader elimination in RFID systems," Second Annual IEEE Communications Society Conference on SECON, Santa Clara, California, USA, Sept. 2005.

[5] ETSI EN 302 208-1 V1.1.1 (2004-09), [Online] www.etsi.org.

[6] EPCglobal, "EPC radio-frequency identity protocols class-1 generation-2 UHF RFID protocol for communications at $860 \mathrm{MHz}-960 \mathrm{MHz}$ version 1.0.9," EPCglobal Standard Specification, 2004.

[7] K. S. Leong, M. L. Ng, P. H. Cole, "The reader collision problem in RFID systems," IEEE 2005 International Symposium on MAPE, Beijing, China, 8-12 Aug. 2005.

[8] K. S. Leong, M. L. Ng, P. H. Cole, "Positioning Analysis of Multiple Antennas in a Dense RFID Reader Environment," paper submitted for publication. 